\title{
Autosomal recessive cerebellar ataxia with late-onset spasticity
}

INSERM

\section{Source}

INSERM. (1999). Orphanet: an online rare disease and orphan drug data base. Autosomal recessive cerebellar ataxia with late-onset spasticity. ORPHA:352641

Autosomal recessive cerebellar ataxia with late-onset spasticity is a rare, genetic neurodeg enerative disease characterized by childhood or adolescent-onset of cerebellar ataxia with dysarthria which slowly progresses and associates pyramidal signs, including lower limb spasticity, brisk reflexes, and Babinski and Hoffman signs. Patients typically present cerebellar ataxia with development of increasing asymmetric spasticity in upper and lower limbs, and variable axonal sensory or sensorimotor neuropathy. Additional heterogeneous features, including pes cavus, scoliolis, and abnormalities of the brain (e.g. cerebral atrophy), may also be associated. 